\title{
KIF1A missense mutations in SPG30, an autosomal recessive spastic paraplegia: distinct phenotypes according to the nature of the mutations
}

\author{
Stephan Klebe ${ }^{1,2,3,4,5,6}$, Alexander Lossos ${ }^{7}$, Hamid Azzedine $e^{1,3,4}$, Emeline Mundwiller ${ }^{1,3,4}$, Ruth Sheffer ${ }^{7}$, \\ Marion Gaussen ${ }^{1,3,4}$, Cecilia Marelli ${ }^{2}$, Magdalena Nawara ${ }^{1,3,4}$, Wassila Carpentier ${ }^{8}$, Vincent Meyer ${ }^{9,10}$, \\ Agnès Rastetter ${ }^{1,3,4,11}$, Elodie Martin ${ }^{1,3,4,11}$, Delphine Bouteiller ${ }^{1,3,4}$, Laurent Orlando ${ }^{1,3,4,11}$, Gabor Gyapay ${ }^{9}$, \\ Khalid H El-Hachimi ${ }^{1,3,4,11}$, Batel Zimmerman ${ }^{7}$, Moriya Gamliel$^{7}$, Adel Misk ${ }^{12}$, Israela Lerer ${ }^{7}$, \\ Alexis Brice B $^{\star 1,2,3,4,6}$, Alexandra Durr ${ }^{1,2,3,4,6}$ and Giovanni Stevanin ${ }^{\star, 1,2,3,4,11}$
}

The hereditary spastic paraplegias (HSPs) are a clinically and genetically heterogeneous group of neurodegenerative diseases characterised by progressive spasticity in the lower limbs. The nosology of autosomal recessive forms is complex as most mapped loci have been identified in only one or a few families and account for only a small percentage of patients. We used next-generation sequencing focused on the SPG30 chromosomal region on chromosome 2 q37.3 in two patients from the original linked family. In addition, wide genome scan and candidate gene analysis were performed in a second family of Palestinian origin. We identified a single homozygous mutation, P.R350G, that was found to cosegregate with the disease in the SPG30 kindred and was absent in 970 control chromosomes while affecting a strongly conserved amino acid at the end of the motor domain of KIF1A. Homozygosity and linkage mapping followed by mutation screening of KIF1A allowed us to identify a second mutation, p.A255V, in the second family. Comparison of the clinical features with the nature of the mutations of all reported KIF1A families, including those reported recently with hereditary sensory and autonomic neuropathy, suggests phenotypegenotype correlations that may help to understand the mechanisms involved in motor neuron degeneration. We have shown that mutations in the KIF1A gene are responsible for SPG30 in two autosomal recessive HSP families. In published families, the nature of the KIF1A mutations seems to be of good predictor of the underlying phenotype and vice versa.

European Journal of Human Genetics (2012) 20, 645-649; doi:10.1038/ejhg.2011.261; published online 18 January 2012

Keywords: hereditary spastic paraplegia; motor neuron disease; hereditary sensory and autonomic neuropathy; next-generation sequencing; SPG30; KIF1A

\section{INTRODUCTION}

The hereditary spastic paraplegias (HSPs) are a clinically and genetically heterogeneous group of neurodegenerative diseases characterised by progressive spasticity in the lower limbs. ${ }^{1}$ The mode of inheritance may be autosomal dominant, autosomal recessive (ARHSP) or $\mathrm{X}$-linked. More than 48 different loci (SPGn) have been mapped so far, and 23 responsible genes identified. The corresponding proteins are often involved in intracellular trafficking or mitochondrial functions. ${ }^{2,3}$

Clinically, one can distinguish between pure and complicated forms of HSP. ${ }^{1,2}$ Pure forms consist of isolated pyramidal signs, such as spasticity, abnormal reflexes (brisk reflexes and Babinski sign) and motor deficit, often associated with sphincter disturbances and deep sensory loss. In the complicated forms of HSP the disease is variably associated with numerous combinations of neurological and extraneurological signs, such as cerebellar ataxia, dysarthria, mental retardation, peripheral neuropathy, optic atrophy, retinitis pigmentosa and/or hearing loss, which may be accompanied by abnormal brain MRI (atrophy of the cortex, cerebellum or corpus callosum, white matter abnormalities, etc).

Mutations in the KIF1A gene (MIM 601255) were very recently described in two different clinically and genetically heterogeneous groups of neurodegenerative diseases. ${ }^{4,5}$ In hereditary sensory and autonomic neuropathy (HSAN), all patients from four families with different origins shared a common homozygous region on chromosome 2q37.3 and carried nonsense mutations of KIF1A. In ARHSP, the KIF1A gene was suspected to be causal in three patients from a single Palestinian consanguineous family following the identification of a missense p.A255V mutation using a whole-exome sequencing strategy combined with homozygosity mapping. ${ }^{4}$

In this study, we show evidence that KIF1A is the causal gene for SPG30, an HSP locus on chromosome 2q37.3, using targeted nextgeneration sequencing (NGS) in the original SPG30 family, ${ }^{6}$ and whole-genome linkage analysis with candidate gene sequencing in a

${ }^{1}$ INSERM, U975, Paris, France; ${ }^{2}$ AP-HP, Hôpital de la Pitié-Salpêtrière, Département de Génétique et Cytogénétique, Centre de Génétique Moléculaire et Chromosomique, Paris, France; ${ }^{3}$ Université Pierre et Marie Curie - Paris 6, UMR_S975, CRICM, Hôpital Pitié-Salpêtrière, Paris, France; ${ }^{4}$ CNRS, UMR_7225, Hôpital de la Pitié-Salpêtrière, Paris, France; ${ }^{5}$ INSERM, AP-HP, Centre d'Investigation Clinique CIC9503, Hôpital de la Pitié-Salpêtrière, Paris, France; ${ }^{6}$ AP-HP, Hôpital de la Pitié-Salpêtrière, Fédération des maladies du système nerveux, Paris, France; ${ }^{7}$ Hadassah-Hebrew University Medical Center, Jerusalem, Israel; ${ }^{8}$ P3S Platform, Hôpital de la Pitié-Salpêtrière, Paris, France; ${ }^{9}$ Genoscope, IG-DSV-CEA, Evry, France; ${ }^{10}$ Genoscope, CEA/INSERM, Paris, France; ${ }^{11}$ Ecole Pratique des Hautes Etudes, Paris, France; ${ }^{12}$ Shaare Zedek Medical Center, Jerusalem, Israel *Correspondence: Dr A Brice or G Stevanin, Institut du Cerveau et de la Moelle épinière, Hôpital de la Pitié-Salpêtrière, 47 boulevard de l'Hôpital 75651 Paris Cedex 13, France. Tel: +33 1572746 80; Fax: +33 1572747 95; E-mail: alexis.brice@upmc.fr (AB) or giovanni.stevanin@upmc.fr (GS)

Received 6 October 2011; revised 14 November 2011; accepted 7 December 2011; published online 18 January 2012 
second family. Clear genotype-phenotype correlations were established based on the clinical features of all KIF1A-mutated patients.

\section{PATIENTS AND METHODS}

\section{Subjects}

Two consanguineous families were included in this study (Figure 1), including the original Algerian SPG30 family (FSP546; ${ }^{6}$ ) and a new family (FSP1079) with seven affected and two unaffected members of Palestinian ancestry with no known genealogical connection with the pedigree of Erlich et al. ${ }^{4}$ All individuals were clinically examined by certified neurologists specialised in movement disorders, using standardised charts, and their blood was taken after written consent was obtained and with the approval of the Local Ethics Committees. Standard protocols were used for DNA preparation.

\section{Genetic studies}

SPG30 exon capture and NGS of family FSP546. Sequencing of all exons of the linkage interval on chromosome 2q37.3 was performed with the use of targeted enrichment and NGS on two members of family FSP546 (patient 9 and carrier parent 1) at the Genoscope facility (Evry, France). To this end, a custom sequence capture array (Roche NimbleGen, Madison, WI, USA) focusing on exons in the region on chromosome 2 between 236902739 and 242575292 bp was designed and used to hybridise shotgun fragment libraries obtained from both selected subjects. Massively parallel sequencing was performed on this enriched library using the Roche 454 GS FLX sequencer (Roche) with titanium reagents. Sequence data were then aligned with the hg18 version of the human genome as a reference. Following enrichment capture of all exons of the SPG30 interval and sequencing in patient 9 and parent 1, a mean of 247394 sequencing reads was obtained with an average length of $312 \mathrm{bp}$, of which $95 \%$ of all reads could be mapped, $67 \%$ of them overlapping the enriched regions (Supplementary Table 1). Out of the total of 783 enriched regions, $88-91 \%$ were covered entirely, and $96 \%$ of targeted bases were covered at least 10 -fold. After PCR duplicates removal, the mean coverage of enriched regions (real coverage) was $73 \times$ (from 67 to $80 \times$ in samples FSP546-1 and FSP546-9, respectively).

Novel identified variants were evaluated for their impact on the encoded protein; conservation across vertebrate species, Caenorhabditis elegans, and Drosophila melanogaster; expression patterns; and potential overlap with known miRNAs.
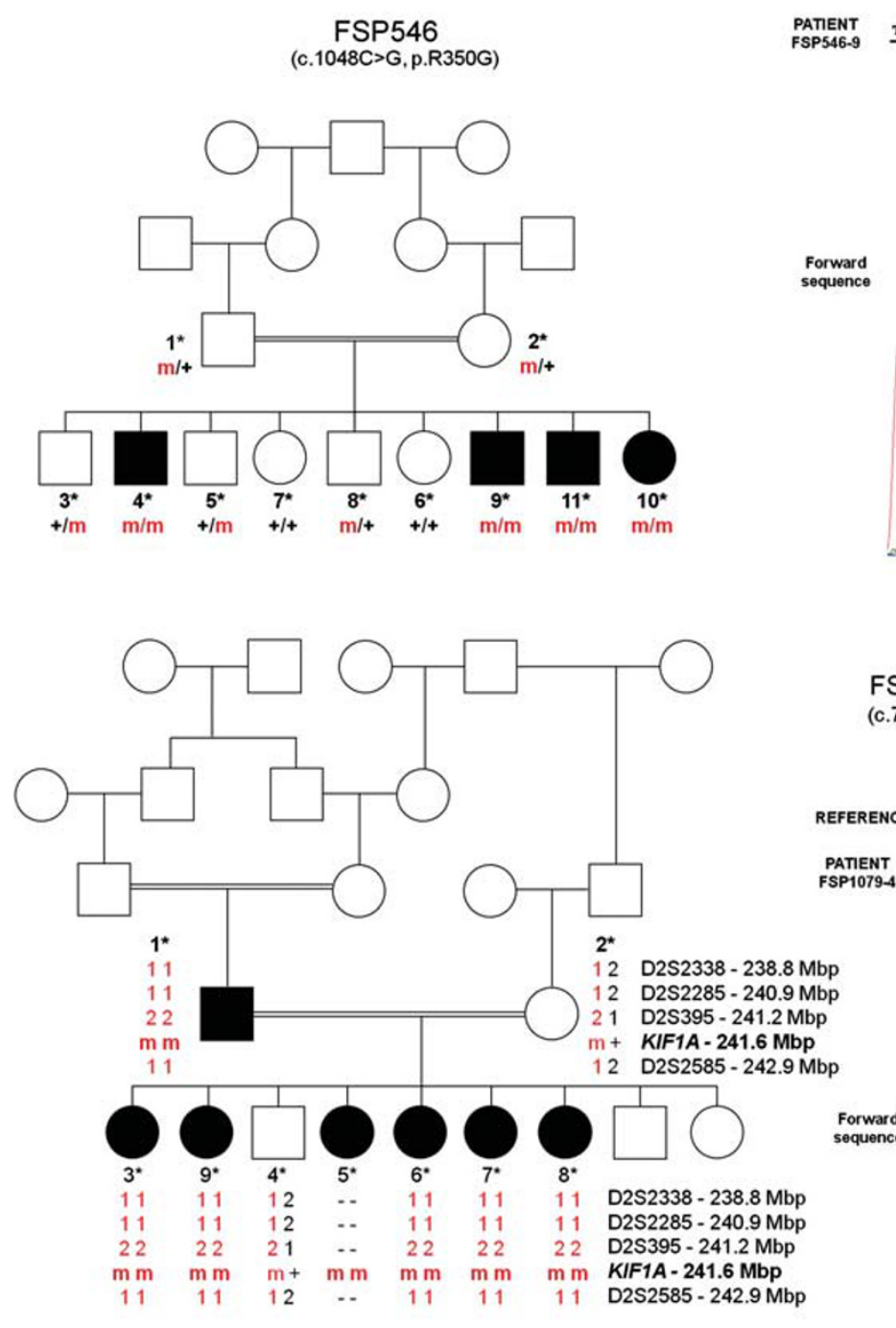

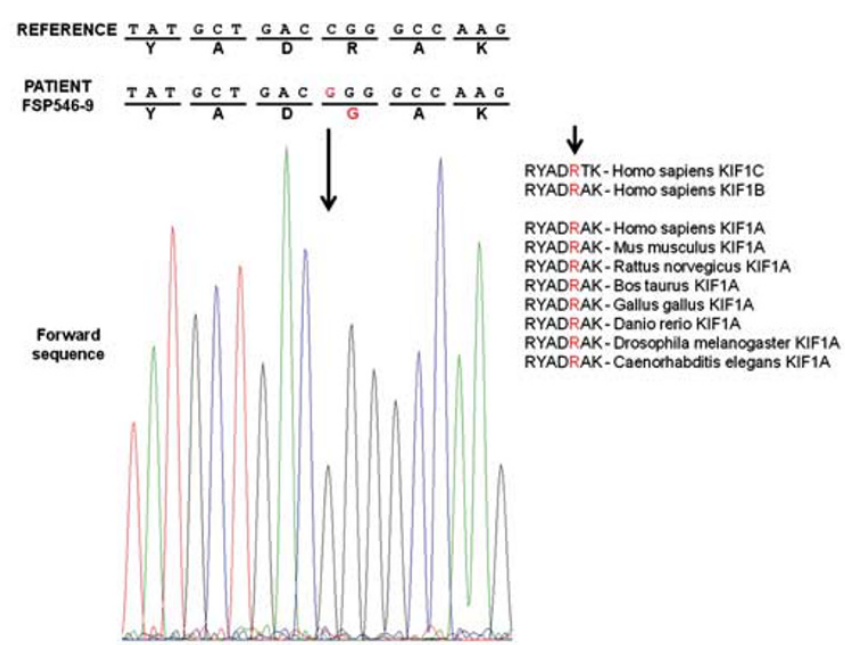

FSP1079 (fsp41)

c.764C>T, p.A255V)

Figure 1 Pedigree of families FSP546 and FSP1079 carrying missense mutations in the KIF1A gene. The code numbers of all sampled individuals are given below the symbols. Black circles (women) and squares (men) indicate affected members. Arrow: index case. Wild type $(+)$ or mutated (m) allelic forms of KIF1A at amino acid 255 (for FSP1079) and 350 (for FSP546). In family FSP1079, the haplotype for four flanking microsatellites is shown. '-' No genotyping results are available. The conservation of the mutated amino acid at positions 255 and 350 in other species is shown to the right of each electropherogram. 


\section{Whole-genome genotyping of family FSP1079}

In the consanguineous Palestinian FSP1079 family, whole-genome genotyping was carried out using the Infinium Human Linkage_12 Genotyping BeadChip (Illumina, San Diego, CA, USA). The BeadChip included 6090 single-nucleotide polymorphism markers with an average gap of $441 \mathrm{~kb}$ and $0.58 \mathrm{cM}$ across the genome. The genotype assignments were determined with Bead Studio genotyping module software (Illumina). Linkage analysis was performed using MERLIN (http://www.sph.umich.edu/csg/abecasis/Merlin/index.html), assuming a fully penetrant recessive disease with similar male-female recombination frequencies and equal allele frequencies. A gene frequency value of 0.0005 was used. A total of 24 additional microsatellite markers were analysed to confirm or exclude non-significantly excluded regions, as described elsewhere. ${ }^{6}$

\section{Sanger sequencing of KIF1A}

In all, 44 amplicons of $\sim 500 \mathrm{bp}$ covering the KIF1A gene were designed using primer couples with melting temperature of $60^{\circ} \mathrm{C}$ (primers available from the authors on request). BigDye chemistry was used for the subsequent Sanger sequencing and the PCR products were resolved in an ABI3730 sequencer according to the manufacturer's instructions (Applied Biosystems, Foster City, CA, USA).

\section{Rearrangement detection}

In order to detect large rearrangements that might affect one gene of the SPG30 interval, two affected individuals, one from each family, were subjected to CGH analysis using the $3 \times 720 \mathrm{~K}$ exon-enriched Nimblegen microarrays in our local facility. In addition, one patient from family FSP1079 was tested using the CYTO_12 Illumina microarray.

\section{RESULTS}

Exon-enriched targeted sequencing of chromosome $2 q 37$ in family FSP546

After initial filtering excluding variants that had fewer than three reads or accounted for $25 \%$ or less of all reads and excluding those reported as polymorphisms in public and local databases, a total of 118 variants mapping to the SPG30 region common to both tested subjects were identified. Only two of these variants were homozygous in the affected patient and heterozygous in the carrier parent. One was in the $3^{\prime}$-UTR of a histone deacetylase 4 (MIM 605314), enzymes responsible for the deacetylation of lysine residues on the $\mathrm{N}$-terminal part of the core histones and then involved in transcription regulation. This variant was not further analysed because of phenotypic features in patients with histone deacetylase 4 mutations (brachydactyly mental retardation syndrome, MIM 600430) and in the corresponding knockout mouse model, which do not overlap with the disease profile of SPG30 patients. The second variant was present in the coding region of KIF1A (c.1048C > G, exon 13 of NM_004321.4) leading to the p.R350G amino-acid change, located in the motor domain of kinesin $1 \mathrm{~A}$ and affecting a highly conserved amino acid (Figure 1). This variant was present at the homozygous state in patients only and was absent in the 970 Caucasian/North African control chromosomes.

\section{Whole-genome genotyping of family FSP1079 and candidate gene analysis}

In family FSP1079 with a pseudo-dominant but autosomal recessive transmission of the disease, whole-genome genotyping revealed a single homozygous region on chromosome $2 \mathrm{q}$, with a multipoint LOD score reaching the maximal expected value of +2.63 from position $251.6 \mathrm{cM}$ to the telomere. This LOD score did not reach significance, due to the pseudo-dominant inheritance of the disease, but overlapped with the SPG30 interval as confirmed using four microsatellite markers (Figure 1). This interval contains the KIF1A gene. Conventional Sanger sequencing of KIF1A revealed the homozygous c.765C $>\mathrm{T}$ (p.A255V) missense mutation in all affected members. This mutation was identical to the one found in a family of similar origin, suggesting a genetic founder effect. ${ }^{4}$ This missense mutation affected a conserved amino acid of the motor domain of the protein and was absent in 184 control chromosomes of Israeli-Arab origin.

\section{Rearrangement detection}

No large rearrangements in the SPG30 candidate interval could be detected by CGH in affected patients of both linked families, using either CYTO_12 (Illumina) or 3x720K (Nimblegen) CGH. This does not exclude the involvement of small rearrangements or inversions.

\section{Clinical features of both families and disease progression in a 5-year follow-up in family FSP546}

In family FSP546 (Figure 1; Table 1), there were nine siblings (six men, three women) born to parents who were first cousins. All 11 members were examined in 2005 and sampled for DNA extraction. ${ }^{6}$ Four of the sibs (three men, one woman) were clinically affected and neurological examination was normal in the remaining children and both parents. The mean age at onset was $17.5 \pm 4$ years (range, 12-21 years). The overall picture was spastic gait with variably associated distal wasting, sensory neuropathy and slight cerebellar ataxia. ${ }^{6}$ By comparison, 5 years after the initial examination of the affected members, ${ }^{6}$ the SPG30 phenotype in family FSP546 shows only a slow progression. This is true for the pyramidal symptoms (spasticity, walking difficulties) and the associated cerebellar signs (dysmetria, saccadic ocular pursuit). All affected members are still able to walk after a disease duration of between 9 and 22 years even if the maximal walking distance is reduced in all patients.

The seven affected members of the Palestinian family FSP1079 (Table 1) have an overall clinical picture of spastic gait with variably associated impaired pin-brick and axonal neuropathy after a disease duration of between 9 and 16 years. Age at onset could be determined in three patients only and ranged from 10 to 39 years.

\section{DISCUSSION}

SPG30 is caused by KIF1A mutations

Several lines of evidence enable us to confirm the KIF1A gene (MIM 601255) as causal for the SPG30 subtype of ARHSP. First, using targeted NGS, we identified only two segregating variants in the original SPG30 family (FSP546). Only one of these was compatible with the HSP phenotype, the p.R350G variant found in KIF1A, which affects a strongly conserved amino acid in the motor domain of kinesin 1A, a specific actor of axonal transport in the nervous system. The mutation was absent in a large set of controls with various geographical backgrounds. Second, in the Palestinian family (FSP1079), whole-genome genotyping revealed a single homozygous region on chromosome $2 \mathrm{q}$ and the presence of the homozygous c.765C $>$ T (p.Ala255Val) missense mutation in all affected members. A genetic founder effect of our family FSP1079 and the family published by Erlich et al ${ }^{4}$ can be presumed, even though it has not yet been tested, as they share the same mutation.

The identification of two mutations in a conserved domain of the KIF1A gene that segregate with the disease in each family, including one mapped significantly, and are located in a single region of homozygosity on chromosome $2 \mathrm{q}$, validate this gene as causal for SPG30. The KIF1A protein belongs to the kinesin-3 family, representing microtubule-dependent proteins that mediate specific and diverse motile processes, including intracellular transport. The KIF1A gene encodes a 1690-amino-acid protein. The p.A255V and p.R350G mutations are localised in the motor domain of the protein. Because 
Table 1 Clinical and paraclinical profiles KIF1A-mutated patients with hereditary spastic paraplegia in our study and in the study of Erlich et al ${ }^{4}$

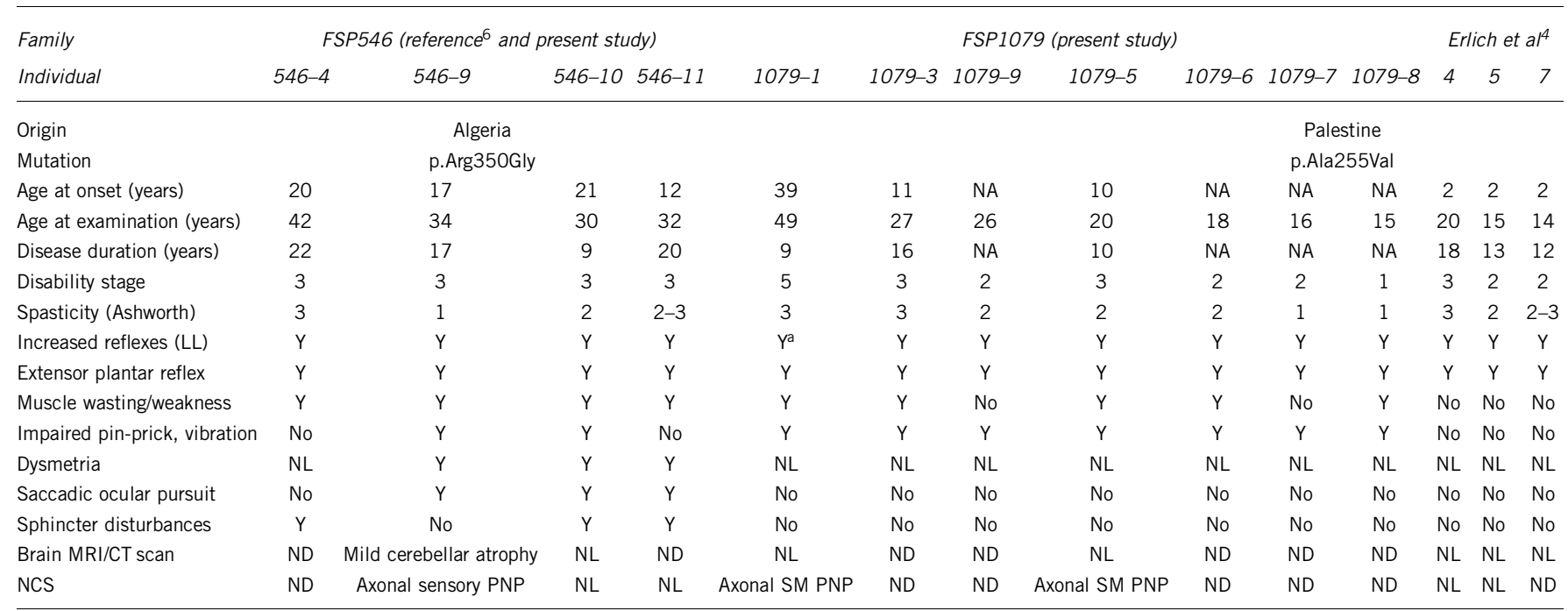

Abbreviations: LL, lower limbs; NA, not available; NCS, nerve conduction study; ND, not done; NL, normal; PNP, peripheral neuropathy; SM, sensory motor; Y, yes.

apattellar, but not at ankle.

of the conservation and localisation of both mutations, a destabilisation of the protein structure is expected that may alter velocity or mobility control. The R350 amino acid in particular is located at the end of the motor domain in close vicinity to the neck linker (aa 352-364) that has an important role in directionality and has mechanochemical implications for the motility of the protein. ${ }^{7-9}$ There is evidence that the KIF1A protein functions as the primary motor for synaptic- and dense-core vesicle transport. ${ }^{10,11}$ In animal models of C. elegans with mutations in the KIF1A ortholog (unc-104), a decreased transport capacity of synaptic vesicle precursors in the axons has been evidenced. ${ }^{11}$ The phenotype of KIF1A knockout mice includes motor and sensory disturbances, a reduction in the density of synaptic vesicles in nerve terminals, and the accumulation of clear vesicles in nerve cell bodies. ${ }^{12}$ In addition, mutations in other kinesinencoding genes, such as KIF5 $A^{13,14}$ and KIF1b (MIM 605995), have been implicated in motor neuron diseases, and intracellular trafficking is a well known mechanism causing the degeneration of the long axonal tracts in such diseases. ${ }^{15}$ In particular, mutations in the KIF5A gene leading to autosomal dominant SPG10 ${ }^{13,14}$ have been shown to reduce the microtubule affinity and/or gliding velocity of kinesin. ${ }^{15}$

\section{SPG30 phenotype}

In the three known HSP families with KIF1A mutation (Table 1), the affected members have a very slowly progressive spastic gait with variably associated distal wasting, peripheral neuropathy in most patients and slight cerebellar ataxia in a few patients. The peripheral neuropathy is reminiscent of the phenotype observed in KIF1A knockout mice. ${ }^{12}$ In family FSP546, the clinical picture was associated with slight cerebellar signs, such as saccadic ocular pursuit, dysmetria and difficulty with tandem standing. The combination of spastic paraparesis with cerebellar symptoms is also a frequent finding in nearly $50 \%$ of ARHSP forms. ${ }^{1}$ In addition, the mild cerebellar involvement, which was found in FSP546 patients, may be overlooked when examining patients.

As in other HSP forms, ${ }^{1}$ the phenotypic presentation varied from one patient to another. Age at onset varied from 2 to 39 years old (mean, 13.6 \pm 11.4 years) but very early ages at onset were exclusively observed in the family published by Erlich et al. ${ }^{4}$ The coexistence of pure and complicated profiles within one form of HSP is well known in SPG4, SPG5, SPG7, SPG10, SPG27 and SPG31 patients. ${ }^{13,16-21}$ Several recently discovered ARHSP forms were initially thought to be pure ARHSP without any cerebellar signs or cerebellar atrophy on brain imaging, a view that was subsequently revised after more families were identified. This was the case for SPG7 (paraplegin) and SPG5 (CYB7B1). ${ }^{16,17,22,23}$

In terms of disease progression, SPG30 differs from other ARHSPs, such as SPG11,24 in having a less severe evolution, as all affected members were still able to walk after disease durations of between 9 and 22 years even if maximal walking distance was reduced in all patients.

\section{Phenotype and genotype correlations in SPG30 and HSAN}

In light of this study and two previous reports dealing with KIF1A mutations in neurodegenerative diseases, ${ }^{4,5}$ we observed that the nature of the KIF1A mutations could predict the phenotype (Table 2). Nonsense mutations, likely leading to a complete loss of function of the protein, cause $\mathrm{HSAN}^{5}$ with major clinical alterations of the peripheral nervous system (PNS). Missense mutations located in the kinesin motor domain of KIF1A are associated predominantly with an upper motor neuron syndrome of the central nervous system (Table 2). However, PNS alterations also occur frequently in HSP patients as peripheral neuropathy was observed in $64 \%$ of the SPG30 patients. How different mutation types in the same gene may differentially alter primary and secondary motor neurons remains unclear but may be related to a residual function of the protein with missense mutation compared with its complete loss of function. These phenotype-genotype correlations have been inferred from seven mutated kindred only and the identification of new KIF1Amutated patients may complicate this picture as it is now the case for SPG10 with the involvement of other types than missense mutations in KIF5A. ${ }^{25}$

In conclusion, we have shown that mutations in the KIF1A gene are responsible for the SPG30 form in two linked autosomal recessive HSP 
Table 2 Clinical characteristics of the known KIF1A-mutated families with HSP or HSAN phenotypic presentation

\begin{tabular}{|c|c|c|}
\hline Disease & $\begin{array}{l}\text { HSP (present study } \\
\text { and references }{ }^{4,6} \text { ) }\end{array}$ & HSAN (reference ${ }^{5}$ ) \\
\hline Number of families & 3 & 4 \\
\hline Number of patients & 14 & 8 \\
\hline Geographical origin & $\begin{array}{l}\text { Algeria, Palestinian } \\
\text { territories }\end{array}$ & $\begin{array}{l}\text { Afghanistan, } \\
\text { Turkey, Belgium }\end{array}$ \\
\hline Mutation & p.R350G; p.A255V & $\begin{array}{l}\text { p.L947Rfs4; } \\
\text { p.S1758QInfs7 }\end{array}$ \\
\hline Age at onset (range in years) & $2-39$ & CG-15 \\
\hline $\begin{array}{l}\text { CNS signs ( } \% \text { of affected } \\
\text { patients with each } \\
\text { sign/symptom) }\end{array}$ & $\begin{array}{l}\text { Spastic paraparesis (100) } \\
\text { Extensor plantar } \\
\text { response (100) } \\
\text { Cerebellar signs }(21)\end{array}$ & $\begin{array}{l}\text { Intellectual } \\
\text { disability/cognitive } \\
\text { impairment (11) }\end{array}$ \\
\hline MRI & $\begin{array}{l}\text { No or mild cerebellar atrophy } \\
\text { (one patient with p.R350G)a }\end{array}$ & Normal \\
\hline $\begin{array}{l}\text { Peripheral neuropathy } \\
\text { (\% of affected patients } \\
\text { with each sign/symptom) }\end{array}$ & $\begin{array}{l}\text { Impaired pinprick } \\
\text { sense (64) } \\
\text { Mild weakness (64) } \\
\text { Decreased SNAP (43) }\end{array}$ & $\begin{array}{l}\text { PNS involvement } \\
\text { in all patients (100) } \\
\text { Painless ulceration (25) } \\
\text { Distal sensory loss (75) } \\
\text { Weakness: mild (62), } \\
\text { severe (38) } \\
\text { Reduced or absent } \\
\text { SNAP (100) }\end{array}$ \\
\hline
\end{tabular}

Abbreviations: CG, congenital; CNS, central nervous system; HSAN, hereditary sensory and autonomic neuropathy; HSP, hereditary spastic paraplegia; PNS, peripheral nervous system; SNAP, sensory nerve action potential.

anformation available in $7 / 14$ patients.

Information available in $6 / 8$ patients.

families with different genetic backgrounds. We suggest that the nature of the KIF1A mutations predicts the underlying phenotype. These phenotype-genotype correlations will have to be confirmed by KIF1A screening efforts in HSP and HSAN families, and it will be important to try to ascertain why different mutations of KIF1A cause markedly different neurological disorders involving predominantly either the CNS or the PNS.

\section{CONFLICT OF INTEREST}

The authors declare no conflict of interest.

\section{ACKNOWLEDGEMENTS}

We are grateful to the DNA and cell bank of the CR-ICM for technical assistance, Drs Bouslam and Nelson for their help and Drs P Bastien, J Melki, V Meiner and M Koenig for patient referral and clinical evaluation. This study was supported financially by grants from the Agence Nationale de la Recherche (ANR SPAX to AD; E-Rare EUROSPA to AB), the Verum Foundation (to $\mathrm{AB}$ ), the Chief Scientist, $\mathrm{MOH}$, Israel/E-Rare 2007 (to $\mathrm{AL}$ ) and the GIS-Rare Diseases Institute (NGS call: ARHSP to AB). SK received fellowships from the post-doctoral programme of the German Academic Exchange Service
(DAAD, Germany), the Verum Foundation and the TWS foundation (Muenster, Germany)'.

1 Depienne C, Stevanin G, Brice A, Durr A: Hereditary spastic paraplegias: an update. Curr Opin Neurol 2007; 20: 674-680.

2 Salinas S, Proukakis C, Crosby A, Warner TT: Hereditary spastic paraplegia: clinical features and pathogenetic mechanisms. Lancet Neurol 2008; 7: 1127-1138.

3 Stevanin G, Ruberg M, Brice A: Recent advances in the genetics of spastic paraplegias. Curr Neurol Neurosci Rep 2008; 8: 198-210.

4 Erlich $\mathrm{Y}$, Edvardson $\mathrm{S}$, Hodges $\mathrm{E}$ et al: Exome sequencing and disease-network analysis of a single family implicate a mutation in KIF1A in hereditary spastic paraparesis. Genome Res 2011; 21: 658-664.

5 Riviere JB, Ramalingam S, Lavastre $V$ et al: KIF1A, an axonal transporter of synaptic vesicles, is mutated in hereditary sensory and autonomic neuropathy type 2. Am J Hum Genet 2011.

6 Klebe S, Azzedine H, Durr A et al: Autosomal recessive spastic paraplegia (SPG30) with mild ataxia and sensory neuropathy maps to chromosome 2q37.3. Brain 2006; 129 : 1456-1462.

7 Al-Bassam J, Cui Y, Klopfenstein D, Carragher BO, Vale RD, Milligan RA: Distinct conformations of the kinesin Unc104 neck regulate a monomer to dimer motor transition. J Cell Biol 2003; 163: 743-753.

8 Case RB, Pierce DW, Hom-Booher N, Hart CL, Vale RD: The directional preference of kinesin motors is specified by an element outside of the motor catalytic domain. Cell 1997; 90: 959-966.

9 Rice S, Lin AW, Safer D et al: A structural change in the kinesin motor protein that drives motility. Nature 1999; 402: 778-784.

10 Lo KY, Kuzmin A, Unger SM, Petersen JD, Silverman MA: KIF1A is the primary anterograde motor protein required for the axonal transport of dense-core vesicles in cultured hippocampal neurons. Neurosci Lett 2011; 491: 168-173.

11 Zahn TR, Angleson JK, MacMorris MA et al: Dense core vesicle dynamics in Caenorhabditis elegans neurons and the role of kinesin UNC-104. Traffic 2004; 5: 544-559.

12 Yonekawa Y, Harada A, Okada Y et al: Defect in synaptic vesicle precursor transport and neuronal cell death in KIF1A motor protein-deficient mice. J Cell Biol 1998; 141: 431-441.

13 Goizet C, Boukhris A, Mundwiller E et al: Complicated forms of autosomal dominant hereditary spastic paraplegia are frequent in SPG10. Hum Mutat 2009; 30: E376E385

14 Reid E, Kloos M, Ashley-Koch A et al: A kinesin heavy chain (KIF5A) mutation in hereditary spastic paraplegia (SPG10). Am J Hum Genet 2002; 71: 1189-1194.

15 Dion PA, Daoud H, Rouleau GA: Genetics of motor neuron disorders: new insights into pathogenic mechanisms. Nat Rev Genet 2009; 10: 769-782.

16 Arnoldi A, Crimella C, Tenderini E et al: Clinical phenotype variability in patients with hereditary spastic paraplegia type 5 associated with CYP7B1 mutations. Clin Genet 2011.

17 De Michele G, De Fusco M, Cavalcanti F et al: A new locus for autosomal recessive hereditary spastic paraplegia maps to chromosome 16q24.3. Am J Hum Genet 1998; 63: 135-139.

18 Goizet C, Boukhris A, Durr A et al: CYP7B1 mutations in pure and complex forms of hereditary spastic paraplegia type 5. Brain 2009; 132: 1589-1600.

19 Heinzlef 0 , Paternotte C, Mahieux F et al: Mapping of a complicated familial spastic paraplegia to locus SPG4 on chromosome 2p. J Med Genet 1998; 35: 89-93.

20 Ribai P, Stevanin G, Bouslam N et al: A new phenotype linked to SPG27 and refinement of the critical region on chromosome. J Neurol 2006; 253: 714-719.

21 Hewamadduma C, McDermott C, Kirby J et al: New pedigrees and novel mutation expand the phenotype of REEP1-associated hereditary spastic paraplegia (HSP). Neurogenetics 2009; 10: 105-110.

22 Elleuch N, Depienne C, Benomar A et al: Mutation analysis of the paraplegin gene (SPG7) in patients with hereditary spastic paraplegia. Neurology 2006; 66: 654-659.

23 Tsaousidou MK, Ouahchi K, Warner TT et al: Sequence alterations within CYP7B1 implicate defective cholesterol homeostasis in motor-neuron degeneration. Am J Hum Genet 2008; 82: 510-515.

24 Stevanin G, Azzedine H, Denora P et al: Mutations in SPG1 1 are frequent in autosomal recessive spastic paraplegia with thin corpus callosum, cognitive decline and lower motor neuron degeneration. Brain 2008; 131: 772-784.

25 Schüle R, Kremer BP, Kassubek J et al: SPG10 is a rare cause of spastic paraplegia in European families. J Neurol Neurosurg Psychiatry 2008; 79: 584-587.

Supplementary Information accompanies the paper on European Journal of Human Genetics website (http://www.nature.com/ejhg) 